\title{
Minimal Founded Semantics for Disjunctive Logic Programs and Deductive Databases*
}

\author{
Filippo Furfaro, Gianluigi Greco and Sergio Greco \\ DEIS \\ Università della Calabria \\ 87030 Rende, Italy \\ \{filippo.furfaro,gianluigi.greco,greco\}@deis.unical.it
}

\begin{abstract}
In this paper, we propose a variant of stable model semantics for disjunctive logic programming and deductive databases. The semantics, called minimal founded, generalizes stable model semantics for normal (i.e. non disjunctive) programs but differs from disjunctive stable model semantics (the extension of stable model semantics for disjunctive programs). Compared with disjunctive stable model semantics, minimal founded semantics seems to be more intuitive, it gives meaning to programs which are meaningless under stable model semantics and is no harder to compute. More specifically, minimal founded semantics differs from stable model semantics only for disjunctive programs having constraint rules or rules working as constraints. We study the expressive power of the semantics and show that for general disjunctive datalog programs it has the same power as disjunctive stable model semantics.
\end{abstract}

KEYWORDS: disjunctive logic programs, disjunctive deductive databases, semantics, minimal models, stable models.

\section{Introduction}

Several different semantics have been proposed for normal and disjunctive logic programs. Stable model semantics, first proposed for normal (i.e. disjunction free) programs, has been subsequently extended to disjunctive programs. For normal programs, stable model semantics has been widely accepted since it captures the intuitive meaning of programs and, for stratified programs it coincides with perfect model semantics which is the standard semantics for this class of programs (?; ?; ?; ?). For positive programs, stable model semantics coincides with minimal model semantics which is the standard semantics for positive disjunctive programs.

* A preliminary version of this paper has been presented at the LPNMR'99 conference (Greco, 1999). Work partially supported by the Murst projects "DataX" and "D2I". The third author is also supported by ISI-CNR. 
For general disjunctive programs several semantics have been proposed. We mention here the generalized closed world assumption (GCWA) (?), the weak generalized closed world assumption (WGCWA) (?; ?), the possible model semantics (?), the perfect model semantics (?), particularly suited to stratified programs, the disjunctive well-founded semantics (?), the disjunctive stable model semantics (?; ?) and the partial stable model semantics (?; ?).

Disjunctive stable model semantics is widely accepted since i) it gives a good intuition of the meaning of programs, ii) for normal programs it coincides with stable model semantics and for stratified (resp. positive) programs it coincides with the perfect (resp. minimal) model semantics. However, disjunctive stable model semantics has some drawbacks. It is defined for a restricted class of programs and there are several reasonable programs which are meaningless, i.e. they do not have stable models.

\section{Motivating examples}

The following examples present some programs whose intuitive meaning is not captured by disjunctive stable model semantics.

Example 1

Consider the following simple disjunctive program $P_{1}$

$$
\begin{aligned}
& a \vee b \vee c \leftarrow \\
& \leftarrow \neg a \\
& \leftarrow \neg b
\end{aligned}
$$

where the second and third rules are constraints, i.e. rules which are satisfied only if the body is false, which can be rewritten into equivalent normal rules. ${ }^{1} P_{1}$ has a unique minimal model $M_{1}=\{a, b\}$ but $M_{1}$ is not stable.

Thus, under stable model semantics the above program is meaningless. However, the intuitive meaning is captured by the unique minimal model since the constraints force more than one atom to be inferred from the disjunctive rule. The next example presents a real life situation that can be easily modeled by means of a disjunctive program.

\section{Example 2}

Consider the Internet structure where every computer in the network makes use of a primary DNS (Domain Name Server) for resolving names associated to IP addresses; moreover if the primary server fails, a secondary (supplementary) DNS is searched. So, an address cannot be resolved if both primary and secondary DNSs are not reachable. An interesting task could be the identification of a minimal set

\footnotetext{
${ }^{1}$ A constraint rule of the form $\leftarrow b_{1}, \ldots, b_{k}$ can be rewritten under total semantics (i.e. a two value semantics where every atom is either true or false) as $p(X) \leftarrow b_{1}, \ldots, b_{k}, \neg p(X)$ where $p$ is a new predicate symbol and $X$ is the list of all distinct variables appearing in the source rule.
} 
of servers that ensures the connectivity of a set of computers. This task can be formalized by the following disjunctive program:

$$
\operatorname{active}\left(D_{1}\right) \vee \operatorname{active}\left(D_{2}\right) \leftarrow \operatorname{dns}\left(C, D_{1}, D_{2}\right)
$$

where active $(D)$ means that $D$ is a working DNS, $\operatorname{dns}\left(C, D_{1}, D_{2}\right)$ means that $C$ is a computer with $D_{1}$ and $D_{2}$ as primary and secondary DNSs. Assuming that $d n s$ is a relation of our database, it is easy to see that this program has minimal (stable) models (under the disjunctive stable model semantics) and that each stable model corresponds to the set of working DNSs.

Now suppose that we are looking for a set of active DNSs containing both $d_{1}$ and $d_{2}$; this situation can be modeled by adding to the program the following constraint:

$$
\begin{aligned}
& \leftarrow \neg \operatorname{active}\left(d_{1}\right) \\
& \leftarrow \neg \operatorname{active}\left(d_{2}\right)
\end{aligned}
$$

Under this hypothesis, if there is a computer $c$ with $d_{1}$ and $d_{2}$ as primary and secondary DNSs (i.e. there is a fact $d n s\left(c, d_{1}, d_{2}\right)$ in the database), the program has a minimal model containing active $\left(d_{1}\right)$ and active $\left(d_{2}\right)$; but this model is not stable. Thus, under stable model semantics this program is meaningless, even though its intuitive meaning is captured by the minimal model.

For a better understanding of this problem, consider now the formalization in terms of logic programming of the 3SAT problem.

\section{Example 3}

The 3SAT problem in which clauses consist of exactly 3 literals can be expressed by the following three rules:

$$
\begin{aligned}
& \operatorname{val}(X, \text { true }) \vee \operatorname{val}(X, \text { false }) \leftarrow \operatorname{var}(X) \\
& \leftarrow \operatorname{val}(X, \operatorname{true}), \operatorname{val}(X, \text { false }) \\
& \operatorname{val}(X, V x) \vee \operatorname{val}(Y, V y) \vee \operatorname{val}(Z, V z) \leftarrow \quad \begin{array}{l}
\operatorname{occur}(C, X, V x), \operatorname{occur}(C, Y, V y), \\
\operatorname{occur}(C, Z, V z)
\end{array}
\end{aligned}
$$

The first two rules state that the value of each literal must be either true or false. In the third rule a predicate $\operatorname{occur}(C, X, V x)$ checks if the literal $X$ occurs in the clause $C$; the value of $V x$ is true (resp. false) if $X$ occurs positively (resp. negatively) in $C$. The set of clauses is described by means of the database predicate occur. For instance, the clause $c_{1}=x_{1} \vee x_{2} \vee \neg x_{3}$ is defined by the three facts occur $\left(c_{1}, x_{1}\right.$, true $)$, occur $\left(c_{1}, x_{2}\right.$, true $)$ and occur $\left(c_{1}, x_{3}\right.$, false $)$. For the sake of simplicity, we are assuming that all clauses consist of exactly three literals. Thus, the third rule above states that for each clause, at least one of its literals must be satisfied.

The above program, for an assigned set of input clauses, has a number of models 
corresponding to all the truth assignments that satisfy all the clauses; so asking for one model is equivalent to solving the $3 \mathrm{SAT}$ problem.

Now suppose that one wants to find a solution in which two variables $x_{1}$ and $x_{2}$ are both true: this situation is modeled as usual by means of the following two constraints:

$$
\begin{aligned}
& \leftarrow \neg \operatorname{val}\left(x_{1}, \text { true }\right) \\
& \leftarrow \neg \operatorname{val}\left(x_{2}, \text { true }\right)
\end{aligned}
$$

If there is no clause in which both $x_{1}$ and $x_{2}$ appear positively, the program still solves the 3SAT problem with constraint; but if there is such a clause then the program has no minimal stable model because the constraint forces more than one atom to be inferred from a disjunctive rule, and the minimal model becomes not stable.

Observe that the first two clauses in the program of the above example can be rewritten into the following normal rules

$$
\begin{aligned}
& \operatorname{val}(X, \text { true }) \leftarrow \operatorname{var}(X), \neg \operatorname{val}(X, \text { false }) \\
& \operatorname{val}(X, \text { false }) \leftarrow \operatorname{var}(X), \neg \operatorname{val}(X, \text { true })
\end{aligned}
$$

since they are used to define a partition of the relation var and the constraint defined by the second rule is used to force exclusive disjunction. Observe also that the constraints $\leftarrow \neg \operatorname{val}\left(x_{1}\right.$, true $)$ and $\leftarrow \neg \operatorname{val}\left(x_{2}\right.$, true $)$ are used to infer, if possible, the atoms $\operatorname{val}\left(x_{1}, \operatorname{true}\right)$ and $\operatorname{val}\left(x_{2}\right.$, true $)$. These constraints cannot be replaced by the two facts $\operatorname{val}\left(x_{1}\right.$, true $) \leftarrow$ and $\operatorname{val}\left(x_{2}\right.$, true $) \leftarrow$ since by doing so we assert that $x_{1}$ and $x_{2}$ are true whereas the constraints are used to force the semantics to infer, if possible, that $x_{1}$ and $x_{2}$ are true.

Intuitively, the problem with stable model semantics is that in some cases the inclusive disjunction is interpreted as exclusive disjunction. This is an old problem first noticed in (?) who proposed an alternative rule, called disjunctive database rule (DDR), to infer negative information. DDR is equivalent to the weak generalized closed world assumption (?), an extension of the generalized closed world assumption proposed in (?).

In this paper we try to conjugate minimality of models and inclusive disjunction by presenting a new semantics, called minimal founded, which overcomes some drawbacks of disjunctive stable model semantics and gives meaning to a larger class of programs by interpreting disjunction in a more liberal way.

\section{Contributions}

The main contributions of the paper are the following: 
- We introduce a semantics for disjunctive programs. The proposed semantics seems to be more intuitive than stable model semantics and it gives meaning to programs which are meaningless under disjunctive stable model semantics.

- We show that the new semantics coincides with disjunctive stable model semantics for normal and positive programs.

- We formally define the expressive power and complexity of the proposed semantics for datalog programs and we show that it has the same expressive power and complexity of disjunctive stable model semantics.

As a consequence, the proposed semantics differs from stable model semantics only for programs containing both disjunctive rules and negation.

Although the full expressive power of disjunctive datalog can be reached by only considering stratified programs, the natural way to express NP problems and problems in the second level of the polynomial hierarchy $\left(\Sigma_{p}^{2}\right.$ and $\Pi_{p}^{2}$ problems) is to use the guess-and-check technique, where the guess part is expressed by means of disjunctive rules and the check part is expressed by means of constraints (i.e. unstratified rules) (?). However, as shown by the previous examples, there are several interesting programs whose intuitive semantics is not captured by stable models. Thus, the problem of defining an intuitive semantics for disjunctive datalog is still an interesting topic.

We point out that the aim of this paper is not the introduction of a more powerful semantics but only the definition of a semantics which gives an intuitive meaning to a larger class of programs. In the same way, disjunctive stable models do not increase the expressive power of stratified disjunctive datalog under the perfect model semantics, but just give semantics to a larger class of programs.

\section{Organization of the paper}

The rest of the paper is organized as follows. Section 2 presents preliminaries on disjunctive datalog, minimal and stable model semantics. Section 3 introduces the minimal founded semantics. Its relation with minimal model semantics and stable model semantics is investigated. Section 4 presents results on the expressive power and complexity of minimal founded semantics. Finally, Section 5 presents our conclusions.

\section{Preliminaries}

A (disjunctive datalog) rule $r$ is a clause of the form

$$
A_{1} \vee \cdots \vee A_{k} \leftarrow B_{1}, \ldots, B_{m}, \neg C_{1}, \ldots, \neg C_{n}, \quad k+m+n>0 .
$$

where $A_{1}, \ldots, A_{k}, B_{1}, \ldots, B_{m}, C_{1}, \ldots, C_{n}$ are atoms of the form $p\left(t_{1}, \cdots, t_{h}\right), p$ is a predicate of arity $h$ and the terms $t_{1}, \cdots, t_{h}$ are either constants or variables. The disjunction $A_{1} \vee \cdots \vee A_{k}$ is the head of $r$, while the conjunction $B_{1}, \ldots, B_{m}, \neg C_{1}, \ldots, \neg C_{n}$ 
is the body of $r$. Moreover, if $k=1$ we say that the rule is normal, i.e. not disjunctive.

We denote by $\operatorname{Head}(r)$ the set $\left\{A_{1}, \ldots, A_{k}\right\}$ of the head atoms, and by $\operatorname{Body}(r)$ the set $\left\{B_{1}, \ldots, B_{m}, \neg C_{1}, \ldots, \neg C_{n}\right\}$ of the body literals. We often use upper-case letters, for example $L$, to denote literals. As usual, a literal is an atom $A$ or a negated atom $\neg A$; in the former case, it is positive, and in the latter negative. Two literals $L_{1}$ and $L_{2}$ are complementary if $L_{1}=A$ and $L_{2}=\neg A$, for some atom $A$. For a literal $L, \neg L$ denotes its complementary literal, and for a set $S$ of literals, $\neg S=\{\neg L \mid L \in S\}$. Moreover, $\operatorname{Body}^{+}(r)$ and $\operatorname{Body}^{-}(r)$ denote the set of positive and negative literals occurring in $\operatorname{Body}(r)$, respectively.

A (disjunctive) logic program is a finite set of rules. A $\neg$-free (resp. $\vee$-free) program is called positive (resp. normal). A term, (resp. an atom, a literal, a rule or a program) is ground if no variables occur in it. In the following we also assume the existence of rules with empty head, called denials, which define constraints ${ }^{2}$, i.e. rules which are satisfied only if the body is false.

The Herbrand Universe $U_{P}$ of a program $P$ is the set of all constants appearing in $P$, and its Herbrand Base $B_{P}$ is the set of all ground atoms constructed from the predicates appearing in $P$ and the constants from $U_{P}$. A rule $r^{\prime}$ is a ground instance of a rule $r$, if $r^{\prime}$ is obtained from $r$ by replacing every variable in $r$ with some constant in $U_{P}$. We denote by ground $(P)$ the set of all ground instances of the rules in $P$.

Given a program $P$ and two predicate symbols (resp. ground atoms) $p$ and $q$, we write $p \rightarrow q$ if there exists a rule where $q$ occurs in the head and $p$ in the body or there exists a predicate (resp. ground atom) $s$ such that $p \rightarrow s$ and $s \rightarrow q$. If $p \rightarrow q$ then we say that $q$ depends on $p$; also we say that $q$ depends on any rule where $p$ occurs in the head. A predicate (resp. ground atom) $p$ is said to be recursive if $p \rightarrow p$.

An interpretation of $P$ is any subset of $B_{P}$. The value of a ground atom $L$ w.r.t. an interpretation $I$, value $_{I}(L)$, is true if $L \in I$ and false otherwise. The value of a ground negated literal $\neg L$ is $\neg$ value $_{I}(L)$. The truth value of a conjunction of ground literals $C=L_{1}, \ldots, L_{n}$ is the minimum over the values of the $L_{i}$, i.e., value $(C)=$ $\min \left(\left\{\right.\right.$ value $\left.\left._{I}\left(L_{i}\right) \mid 1 \leq i \leq n\right\}\right)$, while the value $\operatorname{value}_{I}(D)$ of a disjunction $D=$ $L_{1} \vee \cdots \vee L_{n}$ is their maximum, i.e., value $_{I}(D)=\max \left(\left\{\right.\right.$ value $\left.\left._{I}\left(L_{i}\right) \mid 1 \leq i \leq n\right\}\right)$; if $n=0$, then value $_{I}(C)=$ true and value $(D)=$ false. Finally, a ground rule $r$ is satisfied by $I$ if value $_{I}(\operatorname{Head}(r)) \geq$ value $_{I}(\operatorname{Body}(r))$. Thus, a rule $r$ with empty body is satisfied by $I$ if value $_{I}(\operatorname{Head}(r))=$ true whereas a rule $r^{\prime}$ with empty head is satisfied by $I$ if value $\left._{I}\left(\operatorname{Body}_{(} r^{\prime}\right)\right)=$ false. An interpretation $M$ for $P$ is a model of $P$ if $M$ satisfies each rule in $\operatorname{ground}(P)$. The set of all models of $P$ will be denoted by $\mathcal{M}(P)$.

Minker proposed in (?) a model-theoretic semantics for a positive program $P$, which assigns to $P$ the set of its minimal models $\mathcal{M M}(P)$, where a model $M$

${ }^{2}$ Under total semantics 
for $P$ is minimal, if no proper subset of $M$ is a model for $P$. Accordingly, the program $P=\{a \vee b \leftarrow\}$ has the two minimal models $\{a\}$ and $\{b\}$, i.e. $\mathcal{M M}(P)=$ $\{\{a\},\{b\}\}$. The more general disjunctive stable model semantics also applies to programs with (unstratified) negation (?; ?). Disjunctive stable model semantics generalizes stable model semantics, previously defined for normal programs (?).

\section{Definition 1}

Let $P$ be logic program $P$ and let $I$ be an interpretation for $P, \frac{P}{I}$ denotes the ground positive program derived from $\operatorname{ground}(P)$

1. by removing all rules that contain a negative literal $\neg a$ in the body and $a \in I$, and

2. by removing all negative literals from the remaining rules.

An interpretation $M$ is a (disjunctive) stable model of $P$ if and only if $M \in$ $\mathcal{M M}\left(\frac{P}{M}\right)$.

For general $P$, the stable model semantics assigns to $P$ the set $\mathcal{S M}(P)$ of its stable models. It is well known that stable models are minimal models (i.e. $\mathcal{S M}(P) \subseteq$ $\mathcal{M M}(P))$ and that for negation-free programs minimal and stable model semantics coincide (i.e. $\mathcal{S} \mathcal{M}(P)=\mathcal{M M}(P))$.

An extension of the perfect model semantics for stratified datalog programs to disjunctive programs has been proposed in (?).

A disjunctive datalog program $P$ is said to be locally stratified if there exists a decomposition $S_{1}, \cdots, S_{\omega}$ of the Herbrand base such that for every (ground instance of a) clause

$$
A_{1} \vee \cdots \vee A_{k} \leftarrow B_{1}, \ldots, B_{m}, \neg C_{1}, \ldots, \neg C_{n}
$$

in $P$, there exists an $l$, called level of the clause, so that:

1. $\forall i \leq k \operatorname{stratum}\left(A_{i}\right)=l$,

2. $\forall i \leq m$ stratum $\left(B_{i}\right) \leq l$, and

3. $\forall i \leq n \operatorname{stratum}\left(C_{i}\right)<l$.

where $\operatorname{stratum}(A)=i$ iff $A \in S_{i}$.

The set of clauses in ground $(P)$ having level $i$ (resp. $\leq i$ ) is denoted by $P_{i}$ (resp. $\left.P_{i}^{*}\right)$. Any decomposition of the ground instantiation of a program $P$ is called local stratification of $P$.

The preference order on the models of $P$ is defined as follows: $M \prec N$ iff $M \neq N$ and for each $a \in M-N$ there exists a $b \in N-M$ such that $\operatorname{stratum}(a)>\operatorname{stratum}(b)$. Intuitively, stratum $(a)>\operatorname{stratum}(b)$ means that $a$ has higher priority than $b$.

\section{Definition 2}

Let $P$ be a locally stratified disjunctive datalog program. A model $M$ for $P$ is perfect if there is no model $N$ such that $N \prec M$. The collection of all perfect models of $P$ is denoted by $\mathcal{P} \mathcal{M}(P)$. 
Consider for instance the program consisting of the clause $a \vee b \leftarrow \neg c$. The minimal models are $M_{1}=\{a\}, M_{2}=\{b\}$ and $M_{3}=\{c\}$. Since $\operatorname{stratum}(a)>$ $\operatorname{stratum}(c)$ and $\operatorname{stratum}(b)>\operatorname{stratum}(c)$, we have that $M_{1} \prec M_{3}$ and $M_{2} \prec M_{3}$. Therefore, only $M_{1}$ and $M_{2}$ are perfect models.

Notice that $M \subset N$ implies $M \prec N$; thus, for locally stratified $P, \mathcal{P} \mathcal{M}(P) \subseteq$ $\mathcal{M M}(P)$. For positive $P, \mathcal{M M}(P)=\mathcal{P} \mathcal{M}(P)$ and for stratified $P, \mathcal{P} \mathcal{M}(P)=$ $\mathcal{S M}(P) \subseteq \mathcal{M M}(P)$. The computation of the perfect model semantics of a program $P$ can be done by considering a decomposition $\left(P_{1}, \ldots, P_{\omega}\right)$ of $\operatorname{ground}(P)$ and computing the minimal models of all subprograms, one at time, following the linear order (?; ?; ?). In the decomposition $\left(P_{1}, \ldots, P_{\omega}\right)$, for each $P_{i}$ and for each rule $r$ of $P_{i}$, if $A \in \operatorname{Head}(r)$ and $B \in \operatorname{Body}^{+}(r)$ (resp. $B \in B_{\text {Body }}(r)$ ) then $B$ does not appear in the head of any rule of $P_{j}$ with $j>i$ (resp. $j \geq i$ ).

\section{Minimal Founded Semantics}

In this section we introduce a new semantics for disjunctive programs.

\section{Definition 3}

Let $P$ be a positive disjunctive program and let $M$ be an interpretation. Then,

$$
S_{P}(M)=\left\{a \in B_{P} \mid \exists r \in \operatorname{ground}(P) \wedge a \in \operatorname{Head}(r) \wedge \operatorname{Body}(r) \subseteq M\right\}
$$

$S_{P}^{\omega}(\emptyset)$ denotes the least fixpoint of the operator $S_{P}$.

The operator $S_{P}$ extends the classical immediate consequence operator $T_{P}$ to disjunctive programs by replacing head disjunctions with conjunctions. It is obvious that the operator $S_{P}$, for positive $P$, is monotonic and continuous and, therefore, it admits a least fixpoint.

Definition 4 (Minimal Founded Semantics)

Let $P$ be a disjunctive program and let $M$ be a model for $P$. Then, $M$ is a founded model if it is contained in $S_{P}^{\omega}(\emptyset) . M$ is said to be minimal founded if it is a minimal model of $P$ and it is also founded. The collection of all minimal founded models of $P$ is denoted by $\mathcal{M F}(P)$.

For any program $P$, the set of founded models of $P$ will be denoted by $\mathcal{F}(P)$.

\section{Example 4}

The program $P_{1}$ of Example 1 has a unique minimal model $M_{1}=\{a, b\}$ which is also founded since it is the fixpoint of $S_{\frac{P_{1}}{M_{1}}}$. Observe that the interpretation $N_{1}=\{a, b, c\}$ is a founded model for $P_{1}$ but it is not minimal since $M_{1} \subset N_{1}$.

\section{Fact 1}

Let $P$ be a disjunctive datalog program. Then, $\mathcal{M F}(P) \subseteq \mathcal{M M}(P)$.

Proof. By definition of minimal founded model.

The following example presents a disjunctive program where stable and minimal founded semantics coincide. 
Example 5

Consider the following simple disjunctive program $P_{5}$

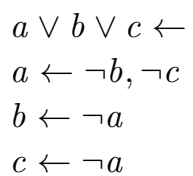

This program has two stable models $M_{5}=\{a\}$ and $N_{5}=\{b, c\}$ which are also minimal founded.

Moreover, for general programs containing both disjunction and negation, stable and minimal founded semantics do not coincide. The relation between the two semantics is given by the following result.

Theorem 1

Let $P$ be a disjunctive program. Then, $\mathcal{S M}(P) \subseteq \mathcal{M} \mathcal{F}(P)$.

Proof. Since stable models are minimal models, we have to show that any stable model $M$ of $P$ is founded, i.e. $M \subseteq S_{P}^{\omega}(\emptyset)$. Since $\frac{P}{M}$ is negation-free, every minimal model of $\frac{P}{M}$ is contained in $S_{\frac{P}{M}}^{\omega}(\emptyset)$. Thus, $M$ is founded and, consequently, $\mathcal{S M}(P) \subseteq$ $\mathcal{M F}(P)$.

Therefore, for every disjunctive program $P, \mathcal{S M}(P) \subseteq \mathcal{M F}(P) \subseteq \mathcal{M M}(P)$. Moreover, as shown by the previous examples, there are programs where the containment is strict, i.e. there are programs, such as the ones presented in the Introduction, having minimal founded models which are not stable.

Corollary 1

Let $P$ be a positive disjunctive datalog program. Then, $\mathcal{M M}(P)=\mathcal{M F}(P)$.

Proof. From Theorem $1 \mathcal{S} \mathcal{M}(P) \subseteq \mathcal{M F}(P)$. Moreover, by definition $\mathcal{M} \mathcal{F}(P) \subseteq$ $\mathcal{M M}(P)$. Since for positive programs $\mathcal{S} \mathcal{M}(P)=\mathcal{M} \mathcal{M}(P)$, we conclude that $\mathcal{M F}(P)=\mathcal{M M}(P)$

The following result states that for disjunction-free programs, stable model semantics and minimal founded semantics coincide.

Proposition 1

Let $P$ be a normal datalog program. Then, $\mathcal{S M}(P)=\mathcal{M} \mathcal{F}(P)$.

Proof. Generally, $\mathcal{S M}(P) \subseteq \mathcal{M} \mathcal{M}(P)$. Thus we have to show that every minimal founded model is also stable. Since for every normal program $P$ and any interpretation $M$ of $P$, the operators $T_{\frac{P}{M}}$ and $S_{\frac{P}{M}}$ coincide, we have that every minimal founded model $M$ of $P$ is equal to $T_{\frac{P}{M}}^{\omega}(\emptyset)$.

The following example presents another case of a program which is meaningless under stable model semantics but has minimal founded models. 
Example 6

Consider the program $P_{6}$

$$
\begin{aligned}
& a \vee b \vee c \leftarrow \\
& a \leftarrow \neg b \\
& b \leftarrow \neg c \\
& c \leftarrow \neg a
\end{aligned}
$$

From the first rule we have that a subset of $\{a, b, c\}$ must be selected whereas the last three rules state that at least two atoms among $a, b$ and $c$ must be true. The program has three minimal founded models, $M_{[6]}=\{a, b\}, N_{[6]}=\{b, c\}$ and $H_{[6]}=\{a, c\}$, but none of them is stable.

It is worth noting that a disjunctive program $P$ may have no, one or several minimal founded models. In the previous example we have presented programs which are meaningless under the stable model semantics which have minimal founded models (those presented in the Introduction) and a program where stable and minimal founded semantics coincide. The following example presents a program which has stable models but the stable and minimal founded semantics do not coincide.

\section{Example 7}

Consider the program $P_{7}$

$$
\begin{aligned}
\text { eat } \vee \text { drink } & \leftarrow \\
\text { eat } & \leftarrow \\
\text { thirsty } & \leftarrow \neg \text { drink }
\end{aligned}
$$

This program has two minimal founded models $M_{0}=\{$ eat, thirsty $\}$ and $N_{[7}=$ $\left\{\right.$ eat, drink\}, but only $M_{7}$ is stable.

We now introduce a different characterization of the minimal founded semantics which permits us to better understand the relationship between stable and minimal founded semantics.

\section{Definition 5}

Let $P$ be a disjunctive program and let $M$ be an interpretation. Then, $P^{M}$ denotes the program derived from ground $(P)$ by deleting for each rule

$$
r: A_{1} \vee \cdots \vee A_{k} \leftarrow B_{1}, \ldots, B_{m}, \neg C_{1}, \ldots, \neg C_{n}
$$

every $A_{i} \notin M$.

\section{Proposition 2}

Let $P$ be a disjunctive program and let $M$ be an interpretation. Then $M \in \mathcal{M F}(P)$ if and only if $M \in \mathcal{M F}\left(P^{M}\right)$. 
Proof. $M$ is a minimal founded model of $P$ iff it is a minimal founded model of $P^{\prime}=\operatorname{ground}(P) . M$ is a minimal model for $P^{\prime}$ if and only if it is a minimal model of $P^{M}$ since we delete from $P^{\prime}$ head atoms which are false in $M$. Moreover, if an atom can be inferred in $\frac{P^{\prime}}{M}$ it can also be inferred in $\frac{P^{M}}{M}$ and vice-versa, i.e. $\mathcal{F}\left(\frac{P^{\prime}}{M}\right)=\mathcal{F}\left(\frac{P^{M}}{M}\right)$. Therefore, $M$ is a minimal founded model for $P^{\prime}$ iff it is a minimal founded model for $P^{M}$.

Observe that the program $P^{M}$ consists of standard rules whose head is not empty and denials (rules with empty head). Thus, in the following we shall denote with $P_{S}^{M}$ the set of standard rules of $P^{M}$ whose head is not empty and with $P_{D}^{M}$ the set of denial rules of $P^{M}$.

Theorem 2

Let $P$ be a disjunctive datalog program and $M$ a minimal model for $P$. Then, $M \in \mathcal{M F}(P)$ if and only $M \in \mathcal{F}\left(P_{S}^{M}\right)$ and $M \models P_{D}^{M}$.

Proof. Clearly $M$ is a minimal founded model for $P$ iff it is a minimal founded model for $P^{\prime}=\operatorname{ground}(P)$.

We first prove that $M \in \mathcal{M F}\left(P^{\prime}\right)$ implies that $M \in \mathcal{F}\left(P_{S}^{M}\right)$ and $M \models P_{D}^{M}$. Let $P^{\prime \prime}$ be the subset of rules in $P^{\prime}$ from which the rules in $P_{D}^{M}$ are derived. Every denial $r: \leftarrow B_{1}, \cdots, B_{m}, \neg C_{1}, \cdots, \neg C_{n}$, derived from a rule $r^{\prime \prime}: A_{1} \vee \cdots \vee A_{k} \leftarrow$ $B_{1}, \cdots, B_{m}, \neg C_{1}, \cdots, \neg C_{n}$, is satisfied in $M$ if and only if $r^{\prime \prime}$ is also satisfied in $M$ because all atoms $A_{1}, \cdots, A_{k}$ are false in $M$. As $P_{S}^{M}=P^{\prime}-P^{\prime \prime}$, if $M$ is a (minimal) founded model for $P^{\prime}$ it is also a founded model for $P_{S}^{M}$ since from the rules in $P^{\prime \prime}$ it is not possible to infer any atom.

We now prove that if $M$ is a minimal model of $P$ such that $M \in \mathcal{F}\left(P_{S}^{M}\right)$, then $M \in \mathcal{M F}\left(P^{\prime}\right)$. As $P_{S}^{M} \subseteq P^{\prime}$, if $M$ is a founded model for $P_{S}^{M}$ and is a minimal model for $P^{\prime}$ it will be a minimal founded model for $P^{\prime}$. It is obvious that if $M$ is a minimal model of $P^{\prime}$ every rule of $P^{\prime}$ is satisfied.

It is important to note that in the ground program there are rules which with respect to a given model act as constraints forcing atoms to be true or false. In the following example we reconsider the program $F_{6}$ of Example 6 containing rules which force the selection of two atoms from the disjunctive rule.

\section{Example 8}

The program $P_{[6]}$ of Example[6] admits three minimal founded models: $M_{6}=\{a, b\}$, $H_{[6]}=\{a, c\}$ and $N_{6]}^{[}=\{b, c\}$. The program $P^{M}\left[\overline{6}=\left(P_{S}^{M}, P_{D}^{M}\right)\right.$ is

$$
\begin{aligned}
& a \vee b \leftarrow \\
& b \leftarrow \\
& \leftarrow \neg a
\end{aligned}
$$

where $P_{S}^{M}$ consists of the first two rules and $P_{D}^{M}$ contains the last rule. The only minimal model for $P^{M}[6]$ is $M_{6}$ this model satisfies $P_{D}^{M^{-6}}$ and is a founded model of $P_{S}^{M}$.6. 
As the program $F_{[6]}$ is symmetric, we have that also $H_{[6]}$ and $N_{[6]}$ are minimal founded model of $P$ [6]

Theorem 2 shows the difference between minimal founded and stable model semantics. In particular, given program $P$ and a minimal model $M$ for $P, M$ is stable if $M$ is a minimal model of $\frac{P_{S}^{M}}{M}$ and $M$ satisfies $P_{D}^{M}$ whereas $M$ is a minimal founded model if $M$ is a model of $\frac{P_{S}^{M}}{M}$ and $M$ satisfies $P_{D}^{M}$. Thus, the main difference between the two semantics is that the stable model semantics asks for minimal models of ground $(P)$ which satisfy the constraints $P_{D}^{M}$ and are also minimal for the subset of standard rules $P_{S}^{M}$, whereas the minimal founded model asks for minimal models of $\operatorname{ground}(P)$ which satisfy the constraints $P_{D}^{M}$ and are founded, i.e. their atoms are derivable from the rules in $P_{S}^{M}$.

It is worth noting that the above result can be very useful in the computation of the semantics of programs. Indeed, during the computation of a model, from the assumption of the falsity of atoms we derive constraints which further restrict the search strategy (?; ?).

\section{Expressive Power and Complexity}

In this section we present some results about the expressive power and the data complexity of minimal founded semantics for disjunctive datalog programs (?; ?; ?). We first introduce some preliminary definitions and notation, and then present our results.

Predicate symbols are partitioned into the two sets of base $(E D B)$ and derived $(I D B)$ predicates. Base predicate symbols correspond to database relations on a countable domain $U$ and do not occur in the rule heads. Derived predicate symbols appear in the head of rules. Possible constants in a program are taken from the domain $U$.

A program $P$ has associated a relational database scheme $D S_{P}=\{r \mid r$ is an EDB predicate symbol of $P\}$, thus EDB predicate symbols are seen as relation symbols. A database $D$ on $D S_{P}$ is a set of finite relations, one for each $r$ in $D S_{P}$, denoted by $D(r)$. The set of all databases on $D S_{P}$ is denoted by $\mathbf{D}_{P}$.

Given a database $D \in \mathbf{D}_{P}, P_{D}$ denotes the following logic program:

$$
P_{D}=P \cup\left\{r(t) \leftarrow \mid r \in D S_{P} \wedge t \in D(r)\right\} .
$$

The Herbrand universe $U_{P_{D}}$ is a finite subset of $U$ and consists of all constants occurring in $P$ or in $D$ (active domain). If $D$ is empty and no constant occurs in $P$, then $U_{P_{D}}$ is assumed to be equal to $\{a\}$, where $a$ is an arbitrary constant in $U$.

\section{Definition 6}

A bound query $Q$ is a pair $\langle P, g\rangle$, where $P$ is a disjunctive program and $g$ is a ground literal (the query goal).

We use $X M$ as generic notation for a generic semantics. The result of a query 
$Q=\langle P, g\rangle$ on an input database $D$ is defined in terms of the $X F$ models of $P_{D}$, by taking either the union of all models (brave or possible inference, $\exists_{X F}$ ) or the intersection (cautious or certain inference, $\forall_{X F}$ ).

\section{Definition 7}

Given a program $P$ and a database $D$, a ground atom $g$ is true, under the brave version of the $X F$ semantics, if there exists an $X F$ model $M$ for $P_{D}$ such that $g \in M$. Analogously, $g$ is true, under the cautious version of the $X F$ semantics, if $g$ is true in every $X F$ model. The set of all queries is denoted by $\mathbf{Q}$.

\section{Definition 8}

Let $Q=\langle P, g\rangle$ be a bound query. Then the database collection of $Q$ w.r.t. the set of $X F$ models is:

(a) under the brave version of semantics, the set of all databases $D$ in $\mathbf{D}_{P}$ such that $g$ is true in $P_{D}$ under the brave version of the $X F$ semantics; this set is denoted by $\operatorname{EXP}_{X F}^{\exists}(Q)$;

(b) under the cautious version of semantics, the set of all databases $D$ in $\mathbf{D}_{P}$ such that $g$ is true in $P_{D}$ under the cautious version of the $X F$ semantics; this set is denoted by $\operatorname{EXP}_{X F}^{\forall}(Q)$.

The expressive power of a given version (either brave or cautious) of the $X F$ semantics is given by the family of the database collections of all possible queries, i.e., $E X P_{X F}^{\exists}[\mathbf{Q}]=\left\{E X P_{X F}^{\exists}(Q) \mid Q \in \mathbf{Q}\right\}$ and $E X P_{X F}^{\forall}[\mathbf{Q}]=\left\{E X P_{X F}^{\forall}(Q) \mid Q \in \mathbf{Q}\right\}$.

The database collection of every query is indeed a generic set of databases. A set D of databases on a database scheme $D S$ with domain $U$ is $(W$-)generic if there exists a finite subset $W$ of $U$ such that for any $D$ in $\mathbf{D}$ and for any isomorphism $\theta$ on relations extending a permutation on $U-W, \theta(D)$ is in $\mathbf{D}$ as well (?; ?) - informally, all constants not in $W$ are not interpreted, and relationships among them are only those explicitly provided by the databases. Note that for a query $Q=\langle P, g\rangle, W$ consists of all constants occurring in $P$ and in $g$. From now on, any generic set of databases will be called a database collection.

Following the data complexity approach of (?; ?) for which the query is assumed to be a constant while the database is the input variable, the expressive power coincides with the complexity class of the problem of recognizing the database collection of each query. The expressive power of each semantics will be compared with database complexity classes, defined as follows. Given a Turing machine complexity class $C$ (for instance $P$ or NP), a relational database scheme $D S$, and a database collection $\mathbf{D}$ on $D S, \mathbf{D}$ is $C$-recognizable if the problem of deciding whether $D$ is in $\mathrm{D}$ is in $C$. The database complexity class $D B-C$ is the family of all $C$-recognizable database collections (for instance, $D B-P$ is the family of all database collections that are recognizable in polynomial time). If the expressive power of a given semantics coincides with a complexity class $D B-C$, we say that the given semantics captures (or expresses all queries in) $D B-C$. 
Recall that the classes $\Sigma_{k}^{P}, \Pi_{k}^{P}$ of the polynomial hierarchy (?) are defined by $\Sigma_{0}^{P}=\mathrm{P}, \Sigma_{i+1}^{P}=\mathrm{NP}^{\Sigma_{i}^{P}}$, and $\Pi_{i}^{P}=\operatorname{co}-\Sigma_{i}^{P}$, for all $i \geq 0$. In particular, $\Pi_{0}^{P}=\mathrm{P}$, $\Sigma_{1}^{P}=\mathrm{NP}$, and $\Pi_{1}^{P}=$ co-NP. Using Fagin's Theorem (?) and its generalization in (?), complexity and second-order definability are linked as follows.

\section{Fact 2}

(?; ?) A database collection $\mathbf{D}$ over a scheme $D S$ is in $D B-\Sigma_{k}^{P}$ (resp. $D B$ $\left.\Pi_{k}^{P}\right), k \geq 1$, iff it is definable by a second-order formula $\left(\exists A_{1}\right)\left(\forall A_{2}\right) \cdots\left(Q_{k} A_{k}\right) \Phi$ (resp. $\left.\left(\forall A_{1}\right)\left(\exists A_{2}\right) \cdots\left(Q_{k} A_{k}\right) \Phi\right)$ on $D S$, where the $A_{i}$ are lists of predicate variables preceded by alternating quantifiers and $\Phi$ is first-order.

The following example shows how a NP problem can be expressed by means of a second order formula and how the formula can be translated into a disjunctive datalog program under minimal founded or stable model semantics.

\section{Example 9}

Consider the graph kernel problem defined as: given a directed graph $G=\langle V, E\rangle$, does there exist a kernel for $G$, i.e. is there a set $S \subseteq V$ of vertices such that both (i) for each $i$ in $V-S$, there exists $j$ in $S$ for which the edge $(j, i)$ is in $E$, and (ii) for each $i, j$ in $S,(i, j)$ is not in $E$ ?

We denote the set of all (finite) directed graphs with $\mathbf{D}_{G}$, the set of all graphs in $\mathbf{D}_{G}$ for which a kernel exists with $\mathbf{D}_{G}^{K}$, and $\overline{\mathbf{D}}_{G}^{K}=\mathbf{D}_{G}-\mathbf{D}_{G}^{K}$. Any graph is represented by a database on the database scheme $B D=\{V, E\}$, where $V$ and $E$ store its vertices and edges, respectively.

Consider the following second-order formula over $B D$ :

$$
\exists S \forall x\{[\neg S(x) \wedge \exists y(S(y) \wedge E(y, x))] \vee[S(x) \wedge \forall y(S(y) \Rightarrow \neg E(y, x))]\}
$$

Note that $V$ supplies the interpretation domain of the formula. It is easy to see that a graph $G$ is in $\mathbf{D}_{G}^{K}$ iff the formula is satisfied by $G$. The above formula can be rewritten in the following equivalent Skolem normal format for existential second order formulas:

$$
\begin{aligned}
& \exists S \forall x_{1}, x_{2} \exists y\left\{\quad\left[\neg S\left(x_{1}\right) \wedge S(y) \wedge E\left(y, x_{1}\right)\right] \vee\left[S\left(x_{1}\right) \wedge \neg S\left(x_{2}\right)\right] \vee\right. \\
& {\left.\left[S\left(x_{1}\right) \wedge S\left(x_{2}\right) \wedge \neg E\left(x_{2}, x_{1}\right)\right]\right\} }
\end{aligned}
$$

This formula is then used to construct the following datalog program:

$$
\begin{aligned}
& \mathrm{r}_{1}: \mathrm{s}(\mathrm{W}) \vee \hat{\mathrm{s}}(\mathrm{W}) \leftarrow \\
& \mathrm{r}_{2}: \leftarrow \mathrm{s}(\mathrm{W}), \hat{\mathrm{s}}(\mathrm{W}) . \\
& \mathrm{r}_{3}: \mathrm{q}\left(\mathrm{X}_{1}, \mathrm{X}_{2}\right) \leftarrow \hat{\mathrm{s}}\left(\mathrm{X}_{1}\right), \mathrm{s}(\mathrm{Y}), \mathrm{e}\left(\mathrm{Y}, \mathrm{X}_{1}\right) . \\
& \mathrm{r}_{4}: \mathrm{q}\left(\mathrm{X}_{1}, \mathrm{X}_{2}\right) \leftarrow \mathrm{s}\left(\mathrm{X}_{1}\right), \hat{\mathrm{s}}\left(\mathrm{X}_{2}\right) . \\
& \mathrm{r}_{5}: \mathrm{q}\left(\mathrm{X}_{1}, \mathrm{X}_{2}\right) \leftarrow \mathrm{s}\left(\mathrm{X}_{1}\right), \mathrm{s}\left(\mathrm{X}_{2}\right), \neg \mathrm{e}\left(\mathrm{X}_{2}, \mathrm{X}_{1}\right) . \\
& \mathrm{r}_{6}: \mathrm{g} \leftarrow \neg \mathrm{q}\left(\mathrm{X}_{1}, \mathrm{X}_{2}\right) .
\end{aligned}
$$

where $v$ and $e$ are EDB predicate symbols and $s$ and $\hat{s}$ are used to define a partition of the database domain (the Herbrand universe). Note that the rules $\left(r_{3}\right)-\left(r_{5}\right)$ implement the three conjunctions in the above Skolem normal form formula. 
Let $G=\langle V, E\rangle$ be a directed graph. A minimal founded (or stable model) is constructed as follows. The first two rules non-deterministically select two disjoint subsets of $V$, say $S$ and $\hat{S}$ respectively. For each $x_{1}$ in $\hat{S}$, if there exists a vertex $y$ in $S$ for which $\left(y, x_{1}\right)$ is in $G$ (i.e. $x_{1}$ is connected to some vertex in $S$ ) then the third rule makes $q\left(x_{1}, x_{2}\right)$ true for every $x_{2}$ in $V$. The fourth rule makes $q\left(x_{1}, x_{2}\right)$ true for each $x_{1}$ in $S$ and for each $x_{2}$ in $\hat{S}$, and the fifth rule makes $q\left(x_{1}, x_{2}\right)$ true if both $x_{1}$ and $x_{2}$ are in $S$ and the edge from $x_{2}$ to $x_{1}$ is not in $G$. Note that $q\left(x_{1}, x_{2}\right)$ is derived to be true for every $x_{1}, x_{2}$ in $V$ iff $S$ and $\hat{S}$ cover $V$ and $S$ is a kernel. But $g$ is false iff for every $x_{1}, x_{2}$ in $V, q\left(x_{1}, x_{2}\right)$ is true; so $g$ is false iff $S$ and $\hat{S}$ cover $V$ and $S$ is a kernel.

For a graph for which a kernel exists, $g$ may be either true or false. Moreover there exists at least one stable model which selects a kernel and, therefore, makes $g$ false. For a graph without kernels, $g$ is always true in every stable model.

It is well known that, under total stable model semantics, disjunctive datalog captures the complexity classes $\Sigma_{2}^{P}$ and $\Pi_{2}^{P}$, respectively, under brave and cautious semantics (?), whereas plain datalog (i.e. datalog with negation and without disjunction) captures the complexity classes NP and coNP, respectively, under brave and cautious semantics (?; ?).

We now present some results on the expressive power and data complexity of the minimal founded semantics.

Theorem 3

Given a disjunctive program $P$, a database $D$ on $D S_{P}$, and an interpretation $M$ for $P_{D}$, deciding whether $M$ is a minimal founded model for $P_{D}$ is coNP-complete.

Proof. Let $M$ be an interpretation and consider the complementary problem $\bar{\Pi}$ : is it true that $M$ is not a strongly founded model? $\bar{\Pi}$ is in NP since we can guess an interpretation $N$ and verify in polynomial time that either (i) $M$ is not a founded model for $P_{D}$ or (ii) $N$ is a model for $P_{D}$ and $N \subset M$. Hence the problem $\Pi$ is in coNP.

Moreover, deciding whether an interpretation $M$ for a positive disjunctive program $P_{D}$ is a minimal model is coNP-complete. Since for positive programs minimal models are also founded, then, deciding whether $M$ is minimal founded is coNP-hard. Therefore, deciding whether $M$ is a minimal founded model for $P_{D}$ is coNP-complete.

Observe that, deciding whether an interpretation $M$ is a stable model for $P_{D}$ is also coNP-complete.

Theorem 4

$E X P_{\mathcal{M F}}^{\forall}[\mathbf{Q}]=D B-\Pi_{2}^{P}$.

Proof We first prove that for any query $Q=\langle P, g\rangle$ in $\mathbf{Q}$, recognizing whether a database $D$ is in $\operatorname{EXP}_{\mathcal{M F}}^{\forall}(Q)$ is in $\Pi_{2}^{P}$. To this end, we consider the complementary problem: is it true that $D$ is not in $\operatorname{EXP}_{\mathcal{M F}}^{\forall}(Q)$ ? Now, $D$ is not in $\operatorname{EXP}_{\mathcal{M F}}^{\forall}(Q)$ iff there exists a minimal founded model $M$ of $P_{D}$ such that $g \notin M$. Following the 
line of the proof of Theorem 5 we can easily see that the latter problem is in $\Sigma_{2}^{P}$. Hence, recognizing whether a database $D$ is in $\operatorname{EXP}_{\mathcal{M F}}^{\forall}(Q)$ is in $\Pi_{2}^{P}$.

Let us now prove that every $\Pi_{2}^{p}$ recognizable database collection $\mathbf{D}$ on a database scheme $D S$ is in $\operatorname{EXP}_{\mathcal{M F}}^{\forall}[\mathbf{Q}]$. By Fact 2] $\mathbf{D}$ is defined by a second order formula of the form $\forall \mathbf{R}^{1} \exists \mathbf{R}^{2} \Phi\left(\mathbf{R}^{1}, \mathbf{R}^{2}\right)$. Using the usual transformation technique, the above formula is equivalent to a second order Skolem form formula $\left(\forall \mathbf{S}^{1}\right)\left(\exists \mathbf{S}^{2}\right) \Gamma\left(\mathbf{S}^{1}, \mathbf{S}^{2}\right)$, where

$$
\Gamma\left(\mathbf{S}^{1}, \mathbf{S}^{2}\right)=(\forall \mathbf{X})(\exists \mathbf{Y})\left(\Theta_{1}\left(\mathbf{S}^{1}, \mathbf{S}^{2}, \mathbf{X}, \mathbf{Y}\right) \vee \ldots \vee \Theta_{k}\left(\mathbf{S}^{1}, \mathbf{S}^{2}, \mathbf{X}, \mathbf{Y}\right)\right),
$$

$\mathbf{S}^{1}$ and $\mathbf{S}^{2}$ are two lists of, respectively, $m_{1}$ and $m_{2}$ predicate symbols, containing all symbols in $\mathbf{R}^{1}$ and $\mathbf{R}^{2}$, respectively. Consider the following program $P$ :

$$
\begin{aligned}
& r_{1}: s_{j}^{1}\left(\mathbf{W}_{j}^{1}\right) \vee \hat{s}_{j}^{1}\left(\mathbf{W}_{j}^{1}\right) \leftarrow \quad\left(1 \leq j \leq m_{1}\right) \\
& r_{2}: s_{j}^{2}\left(\mathbf{W}_{j}^{2}\right) \vee \hat{s}_{j}^{2}\left(\mathbf{W}_{j}^{2}\right) \leftarrow \quad\left(1 \leq j \leq m_{2}\right) \\
& r_{3}: q(\mathbf{X}) \quad \leftarrow \Theta_{i}\left(\mathbf{S}^{1}, \mathbf{S}^{2}, \mathbf{X}, \mathbf{Y}\right) \quad(1 \leq i \leq k) \\
& r_{4}: g \quad \leftarrow \neg q(\mathbf{X}) \text {. } \\
& r_{5}: g \quad \leftarrow s_{j}^{2}\left(\mathbf{W}_{j}^{2}\right), \hat{s}_{j}^{2}\left(\mathbf{W}_{j}^{2}\right) \quad\left(1 \leq j \leq m_{2}\right) \\
& r_{6}: \hat{s}_{j}^{2}\left(\mathbf{W}_{j}^{2}\right) \quad \leftarrow g . \quad\left(1 \leq j \leq m_{2}\right) \\
& r_{7}: s_{j}^{2}\left(\mathbf{W}_{j}^{2}\right) \leftarrow g \quad \leftarrow \quad\left(1 \leq j \leq m_{2}\right)
\end{aligned}
$$

where, intuitively, $\hat{s}_{j}^{1}\left(\mathbf{W}_{j}^{1}\right)$ corresponds to $\neg s_{j}^{1}\left(\mathbf{W}_{j}^{1}\right), \quad \hat{s}_{j}^{2}\left(\mathbf{W}_{j}^{2}\right)$ corresponds to $\neg s_{j}^{2}\left(\mathbf{W}_{j}^{2}\right)$ and the rules of group $r_{3}$ defining $q$ are used to implement the disjunction of the above second order formula. Observe that the guesses defined by the rules in the groups $r_{1}$ and $r_{2}$ are used in the rules in the group $r_{3}$ defining the predicate $q$ and that the rules in the groups $r_{5}, r_{6}$ and $r_{6}$ force $g$ to be false. Now it is easy to show that the formula $\left(\forall \mathbf{S}^{1}\right)\left(\exists \mathbf{S}^{2}\right) \Gamma\left(\mathbf{S}^{1}, \mathbf{S}^{2}\right)$ is valid if $g$ is false in all minimal founded models of $P$ (if $g$ is true the last two sets of rules make the second group of rules false).

Theorem 5

$\operatorname{EXP} P_{\mathcal{M F}}^{\exists}[\mathbf{Q}]=D B-\Sigma_{2}^{P}$.

Proof. We first prove that for any query $Q=\langle P, g\rangle$ in $\mathbf{Q}$, recognizing whether a database $D$ is in $\operatorname{EXP}_{\mathcal{M F}_{\mathcal{F}}}^{\exists}(Q)$ is in $\Sigma_{2}^{P} . D$ is in $\operatorname{EXP}_{\mathcal{M F}}^{\exists}(Q)$ iff there exists a minimal founded model $M$ of $P_{D}$ such that $g \in M$. To check this, we may guess an interpretation $M$ of $P_{D}$ and verify that $M$ is a minimal founded model of $P_{D}$. The guess of the interpretation $M$ is polynomial time. To check that $M$ is minimal founded we can ask an $N P$ oracle. Therefore, recognizing whether a database $D$ is in $\operatorname{EXP}_{\mathcal{M F}}^{\exists}(Q)$ is in $\Sigma_{2}^{P}$.

Let us now prove that every $\Sigma_{2}^{p}$ recognizable database collection $\mathbf{D}^{\prime}$ on a database

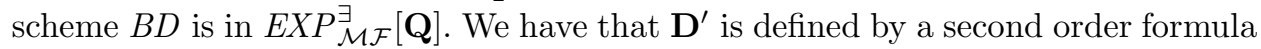
of the form $\exists \mathbf{R}^{1} \forall \mathbf{R}^{2} \Phi^{\prime}\left(\mathbf{R}^{1}, \mathbf{R}^{2}\right)$. By setting $\Phi\left(\mathbf{R}^{1}, \mathbf{R}^{2}\right)=\neg \Phi^{\prime}\left(\mathbf{R}^{1}, \mathbf{R}^{2}\right)$, we have that the formula $\forall \mathbf{R}^{1} \exists \mathbf{R}^{2} \Phi\left(\mathbf{R}^{1}, \mathbf{R}^{2}\right)$ defines the database collection $\mathbf{D}$, where $\mathbf{D}=$ $\mathbf{D}_{B D}-\mathbf{D}^{\prime}$ and $\mathbf{D}_{B D}$ is the set of all databases on $B D$. Consider the program $P$ and the query $Q=\langle P, \neg g\rangle$ in the proof of Theorem 4 In it we have shown that a 
database $D$ in $\mathbf{D}_{B D}$ is in $\mathbf{D}$ iff $D$ is in $E X P_{\mathcal{M F}}^{\forall}(Q)$; hence a database $D$ in $\mathbf{D}_{B D}$ is in $\mathbf{D}^{\prime}$ iff $D$ is not in $\operatorname{EXP}_{\mathcal{M F}}^{\forall}(Q)$. But $D$ is not in $\operatorname{EXP}_{\mathcal{M F}}^{\forall}(Q)$ iff there exists some stable model $M$ for which $g$ is in $M$. It follows that $\mathbf{D}^{\prime}=\operatorname{EXP}_{\mathcal{M F}}^{\exists}\left(Q^{\prime}\right)$ where $Q^{\prime}=\langle P, g\rangle$.

Therefore, the expressive power of disjunctive datalog under minimal founded and stable model semantics is the same.

Data complexity is usually closely tied to expressive power and, in particular, it provides an upper bound for the expressive power (?).

In this section we have shown that minimal founded semantics is complete for the second level of the polynomial hierarchy. For the stable model semantics it has been shown that for the class of head-cycle-free (hcf) the computation of a model selected nondeterministically can be done in polynomial time and checking if a ground atom belongs to a minimal model (resp. all minimal models) is complete for the first level of the polynomial hierarchy, i.e. NP-complete (resp. coNP-complete) (?). This result does not immediately apply to the minimal founded semantics since there could be rules which could force the selection of more than one atom appearing in the head of a rule. We conjecture that we have the same results for the class of head-cycle-free programs where constraints do not force the selection of more than one atom from the head of disjunctive rules. It is possible to identify a syntactic class consisting of hcf programs where after the rewriting of every ground constraint $\leftarrow B(X)$ in $P$ with a rule $p(X) \leftarrow B(X), \neg p(X)$, there is no recursive atom $A$ in $\operatorname{ground}(P)$ depending on itself through an odd number of negations. The formal proof of this is outside the scope of this paper, and it could be investigated in some future work. Another interesting problem to be investigated in the future could be the syntactic characterization of programs for which stable and strongly founded models coincide. Clearly, this class contains positive and normal programs and programs where head disjunctions are forced to be exclusive by constraints.

\section{Conclusion}

The semantics proposed in this paper is essentially a variant of stable model semantics for normal programs. The aim of our proposal is the solution of some drawbacks of disjunctive stable model semantics which, in some cases, interpret inclusive disjunction as exclusive disjunction.

As disjunction is not interpreted as exclusive, the proposed semantics is not invariant if rules which are subsumed by other rules (under stable model semantics) are removed from the program; for instance, the first rule in the program of Example 7 can be deleted under stable model semantics as it is subsumed by the second rule, whereas under the minimal founded model semantics it cannot be deleted.

Several questions which need further investigation have been left open. For in-

stance, further research could be devoted to i) the identification of fragments of disjunctive datalog for which one minimal founded model can be computed in 
polynomial time; ii) the use of two different types of disjunctive rule (inclusive disjunction and exclusive disjunction), iii) the investigation of abstract properties for disjunctive datalog under minimal founded semantics (?).

\section{Acknowledgement}

The authors are grateful to the anonymous referees for their useful comments and suggestions. 